\title{
INTEGRATED REGION-BASED IMAGE RETRIEVAL
}




\title{
THE KLUWER INTERNATIONAL SERIES ON INFORMATION RETRIEVAL
}

\author{
Series Editor \\ W. Bruce Croft \\ University of Massachusetts, Amherst
}

Also in the Series:

MULTIMEDIA INFORMATION RETRIEVAL: Content-Based Information Retrieval from Large Text and Audio Databases, by Peter Schäuble; ISBN: 0-7923-9899-8

INFORMATION RETRIEVAL SYSTEMS: Theory and Implementation, by Gerald Kowalski; ISBN: 0-7923-9926-9

CROSS-LANGUAGE INFORMATION RETRIEVAL, edited by Gregory Grefenstette; ISBN: 0-7923-8122-X

TEXT RETRIEVAL AND FILTERING: Analytic Models of Performance, by Robert $M$. Losee; ISBN: 0-7923-8177-7

INFORMATION RETRIEVAL: UNCERTAINTY AND LOGICS: Advanced Models for the Representation and Retrieval of Information, by Fabio Crestani, Mounia Lalmas, and Cornelis Joost van Rijsbergen; ISBN: 0-7923-8302-8

DOCUMENT COMPUTING: Technologies for Managing Electronic Document Collections, by Ross Wilkinson, Timothy Arnold-Moore, Michael Fuller, Ron Sacks-Davis, James Thom, and Justin Zobel; ISBN: 0-7923-8357-5

AUTOMATIC INDEXING AND ABSTRACTING OF DOCUMENT TEXTS, by MarieFrancine Moens; ISBN 0-7923-7793-1

ADVANCES IN INFORMATIONAL RETRIEVAL: Recent Research from the Center for Intelligent Information Retrieval, by $W$. Bruce Croft; ISBN 0-7923-7812-1

INFORMATION RETRIEVAL SYSTEMS: Theory and Implementation, Second Edition, by Gerald J. Kowalski and Mark T. Maybury; ISBN: 0-7923-7924-1

PERSPECTIVES ON CONTENT-BASED MULTIMEDIA SYSTEMS, by Jian Kang $\mathrm{Wu}$; Mohan S. Kankanhalli;Joo-Hwee Lim;Dezhong Hong; ISBN: 0-7923-7944-6

MINING THE WORLD WIDE WEB: An Information Search Approach, by George Chang, Marcus J. Healey, James A. M. McHugh, Jason T. L. Wang; ISBN: 07923-7349-9

INTEGRATED REGION-BASED IMAGE RETRIEVAL, by James Z. Wang; ISBN: 07923-7350-2 


\title{
INTEGRATED REGION-BASED IMAGE RETRIEVAL
}

\author{
by \\ James Z. Wang \\ The Pennsylvania State University
}

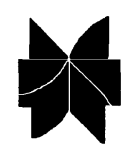

SPRINGER SCIENCE+BUSINESS MEDIA, LLC 


\section{Library of Congress Cataloging-in-Publication Data}

Wang, James Z., 1972-

Integrated region-based image retrieval / James Z. Wang.

p. $\mathrm{cm}$. -- (The Kluwer international series on information retrieval ; 11) Includes bibliographical references.
ISBN 978-1-4613-5655-4
ISBN 978-1-4615-1641-5 (eBook)

DOI 10.1007/978-1-4615-1641-5

1. Optical storage devices. 2. Image processing--Digital techniques. 3. Database management. I. Title. II. Series.

TA1635.W37 2001

006.4 '2--dc21

2001020367

Copyright (C) 2001 by Springer Science+Business Media New York

Originally published by Kluwer Academic Publishers, New York in 2001

Softcover reprint of the hardcover 1st edition 2001

All rights reserved. No part of this publication may be reproduced, stored in a retrieval system or transmitted in any form or by any means, mechanical, photo-copying, recording, or otherwise, without the prior written permission of the publisher, Springer Science+Business Media, LLC

Printed on acid-free paper. 
To my parents 


\section{Contents}

Preface $\quad x i$

Acknowledgments xiii

1. INTRODUCTION 1

1. Text-based image retrieval 2

2. Content-based image retrieval 3

3. Applications of CBIR 3

3.1. Biomedical applications 3

3.2. Web-related applications $\quad 6$

3.3. Other applications $\quad 7$

4. Summary of our work $\quad 7$

4.1. Semantics-sensitive image retrieval 8

4.2. Image classification 9

4.3. Integrated Region Matching distance $\quad 10$

4.4. Applications of the methods 12

5. Structure of the book 12

6. Summary 15

2. BACKGROUND 17

1. Introduction 17

2. Content-based image retrieval 17

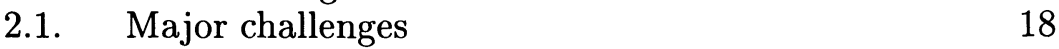

2.2. Previous work 24

2.3. $\quad$ CBIR for biomedical image databases 33

3. Image semantic classification 34

3.1. Semantic classification for photographs 34

3.2. Medical image classification 36

$\begin{array}{ll}\text { 4. Summary } & 37\end{array}$ 
3. WAVELETS 39

1. Introduction 39

2. Fourier transform 40

3. Wavelet transform 41

3.1. Haar wavelet transform 41

3.2. Daubechies' wavelet transform 42

4. Applications of wavelets 46

5. Summary 48

4. STATISTICAL CLUSTERING AND CLASSIFICATION 49

1. Introduction 49

2. Artificial intelligence and machine learning 50

3. Statistical clustering 51

3.1. The k-means algorithm 51

3.2. The TSVQ algorithm 53

4. Statistical classification $\quad 55$

4.1. The CART algorithm $\quad 55$

5. Summary 60

5. WAVELET-BASED IMAGE INDEXING AND SEARCHING 63

1. Introduction 63

2. Preprocessing 64

2.1. Scale normalization $\quad 64$

2.2. Color space normalization 65

3. Multiresolution indexing $\quad 65$

3.1. Color layout $\quad 66$

3.2. Indexing with the Haar wavelet 66

3.3. Overview of WBIIS 67

4. The indexing algorithm 68

5. The matching algorithm $\quad 70$

5.1. Fully-specified query matching $\quad 70$

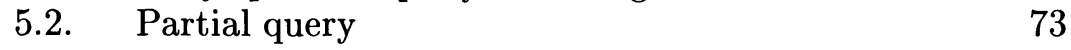

\begin{tabular}{ll} 
6. & Performance \\
\hline
\end{tabular}

7. Limitations 83

8. Summary 84 
6. SEMANTICS-SENSITIVE INTEGRATED MATCHING 85

1. Introduction 85

2. Overview 86

3. Image segmentation $\quad 86$

4. Image classification $\quad 90$

4.1. Textured vs. non-textured images 90

4.2. Graph vs. photograph images 92

5. The similarity metric $\quad 93$

5.1. Integrated region matching $\quad 93$

5.2. Distance between regions 98

6. System for biomedical image databases 101

6.1. $\quad$ Feature extraction 102

6.2. Wavelet-based progressive transmission 102

7. Clustering for large databases 103

8. Summary 104

7. IMAGE CLASSIFICATION BY IMAGE MATCHING 105

1. Introduction 105

2. Industrial solutions 106

3. Related work in academia 106

4. System for screening objectionable images 107

4.1. Moments 108

4.2. The algorithm 109

4.3. Evaluation 113

5. Classifying objectionable websites 114

5.1. The algorithm 115

5.2. Statistical classification process for websites 116

5.3. Limitations 121

5.4. Evaluation 121

6. Summary 122

8. EVALUATION 123

1. Introduction 123

2. Overview 123

3. Data sets 124

3.1. The COREL data set 124

3.2. Pathology data set 124

4. Query interfaces 125

4.1. Web access interface 125

4.2. JAVA drawing interface 126

4.3. External query interface 127

4.4. Progressive browsing 128

5. Characteristics of IRM 128 
6. Accuracy 129

6.1. Picture libraries 131

6.2. Systematic evaluation 136

6.3. Biomedical image databases 144

7. Robustness 145

7.1. Intensity variation 147

7.2. Sharpness variation 148

7.3. Color distortions 148

7.4. Other intentional distortions 149

7.5. Cropping and scaling 150

7.6. Shifting 150

7.7. Rotation 151

8. Speed 152

9. Summary 154

9. CONCLUSIONS AND FUTURE WORK 159

$\begin{array}{ll}\text { 1. Summary } & 159\end{array}$

2. Limitations 160

3. Areas of future work 161

$\begin{array}{ll}\text { References } & 165\end{array}$

Index 


\section{Preface}

Content-based image retrieval is the set of techniques for retrieving relevant images from an image database on the basis of automaticallyderived image features. The need for efficient content-based image retrieval has increased tremendously in many application areas such as biomedicine, the military, commerce, education, and Web image classification and searching. In the biomedical domain, content-based image retrieval can be used in patient digital libraries, clinical diagnosis, searching of 2-D electrophoresis gels, and pathology slides.

I started my work on content-based image retrieval in 1995 when I was with Stanford University. The project was initiated by the Stanford University Libraries and later funded by a research grant from the National Science Foundation. The goal was to design and implement a computer system capable of indexing and retrieving large collections of digitized multimedia data available in the libraries based on the media contents. At the time, it seemed reasonable to me that I should discover the solution to the image retrieval problem during the project. Experience has certainly demonstrated how far we are as yet from solving this basic problem.

CBIR for general-purpose image databases is a highly challenging problem because of the large size of the database, the difficulty of understanding images, both by people and computers, the difficulty of formulating a query, and the problem of evaluating the results. The objectives of this book are to introduce the fundamental problems, to review a collection of selected and well-tested methods, and to introduce our work in this rapidly developing research field.

We designed a content-based image retrieval system with waveletbased feature extraction, semantics classification, and integrated region matching (IRM). An image in the database, or a portion of an image, is represented by a set of regions, roughly corresponding to ob- 
jects, which are characterized by color, texture, shape, and location. The system classifies images into semantic categories, such as texturednontextured, objectionable-benign, or graph-photograph. The categorization enhances retrieval by permitting semantically-adaptive searching methods and narrowing down the searching range in a database. A measure for the overall similarity between images is developed as a region-matching scheme that integrates properties of all the regions in the images. Compared with retrieval based on individual regions, the overall similarity approach reduces the adverse effect of inaccurate segmentation, helps to clarify the semantics of a particular region, and enables a simple querying interface for region-based image retrieval systems.

We built an experimental image retrieval system, the SIMPLIcity (Semantics-sensitive Integrated Matching for Picture LIbraries) system, to validate these methods on various image databases, including a database of about 200,000 general-purpose images and a database of more than 70,000 pathology images. We have shown that our methods perform much better and much faster than existing methods. The system is exceptionally robust to image alterations such as intensity variation, sharpness variation, intentional distortions, cropping, shifting, and rotation. These features are important to biomedical image databases because visual features in the query image are not exactly the same as the visual features in the images in the database. The work has also been applied to the classification of on-line images and web sites. 


\section{Acknowledgments}

This work would not have been possible without the guidance and advice of my dissertation advisor Gio Wiederhold. He has led me to new areas of research, pointed me to interesting research problems, and offered me substantial encouragement. Gio has cultivated a creative atmosphere and provided me with unconditional support.

I would like to thank Dennis A. Hejhal for introducing me to the excitement of conducting scientific research, and for being everlastingly supportive during the past nine years. I would like to thank Martin A. Fischler and Oscar Firschein for inspiring me with the fascinating field of image understanding, and encouraging me. Discussions with Desmond Chan, Shih-Fu Chang, Eldar Giladi, Robert M. Gray, Yoshi Hara, Kyoji Hirata, Xiaoming Huo, Yvan Leclerc, Quang-Tuan Luong, Thomas P. Minka, Wayne Niblack, Richard Olshen, Dragutin Petkovic, Donald Regula, Xin Wei Sha, Michael Walker, and Tong Zhang have been very helpful in different stages of my research. Special thanks goes to Russ B. Altman, W. Bruce Croft, Oscar Firschein, Hector GarciaMolina, Rosalind W. Picard, Mu-Tao Wang, Stephen T.C. Wong, and anonymous reviewers, who provided numerous constructive comments to the manuscript and its related publications.

I would also like to thank my friends in the Stanford Database Group, the Stanford Biomedical Informatics Group, the Stanford Mathematics Department, the Perception Research Group at SRI International, the QBIC Group at the IBM Almaden Research Center, and the School of Information Sciences and Technology and the Department of Computer Science and Engineering at the Pennsylvania State University for their generous help.

My wife $\mathrm{Jia} \mathrm{Li}$ is the most essential contributor to my success and my well-being. Her talents and professional expertise in statistics, information theory, and image processing have enlightened me numerous times 
throughout my research. We have coauthored several publications and experimental systems.

My work was funded primarily by a research grant from the National Science Foundation's Digital Libraries initiative and a research fund from the Stanford University Libraries. I have also received support from IBM Almaden Research Center, NEC Research Lab, SRI International, Stanford Computer Science Department, Stanford Mathematics Department, Stanford Biomedical Informatics, The Pennsylvania State University, and the PNC Foundation. I am truly grateful for the support.

Finally, I acknowledge the Institute for Electrical and Electronic Engineers (IEEE) for their generous permission to use material published in their Transactions and conference proceedings in this book as detailed in specific citations in the text. I would like to thank Scott E. Delman and Melissa Fearon, the editor and editorial assistant at Kluwer Academic Publishers, for making the publication of this book go smoothly. 